\title{
A GENERALIZATION OF GELFAND-MAZUR THEOREM
}

\section{SUNG GUEN KIM}

\author{
Department of Mathematics \\ Pohang Institute of Science and Technology \\ P.O. Box 215 \\ Pohang, 790-600 Korea
}

(Received August 12, 1991 and in revised form November 19, 1991)

ABSTRACT. In this paper, we show that if $A$ is a unital semisimple complex Banach algebra with only the trivial idempotents and if $\sigma_{A}(x)$ is countable for each $x \in \operatorname{Fr}(G(A))$, then $A \cong \mathrm{C}$, this generalizes the Gelfand-Mazur theorem.

\section{INTRODUCTION.}

In this paper, we show that if $A$ is a unital semisimple complex Banach algebra with only the trivial idempotents and if $\sigma_{A}(x)$ is countable for each $x \in \operatorname{Fr}(G(A))$, then $A \cong \mathrm{C}$; this generalizes the Gelfand-Mazur theorem. Throughout this paper we assume that all algebras are over the complex field. We denote by $G(A)$ the set of all invertible elements in $A$ and by $\sigma_{A}(x)$ the spectrum of $x$ in $A$. Let $\rho_{A}(x)$ denote the spectral radius of $x$ in $A$. From the theory of Banach algebras, we know that $\sigma_{A}(x)$ is a nonempty, compact subset of the complex numbers, that $\rho_{A}(x)=\lim _{n}\left\|x^{n}\right\|^{1 / n}$, and that $G(A)$ is an open set in $A$. We denote by $\operatorname{Rad}(A)$ the radical of $A$ and by $\operatorname{Fr}(G(A))$ the boundary of $G(A)$ in $A$. From the theory of Banach algebras, we know that $\operatorname{Rad}(A)$ is a closed two sided ideal.

2. MAIN RESULTS.

LEMMA 1. Let $A$ be a unital Banach algebra. Then $\operatorname{Rad}(A)=\left\{x \in A \mid \rho_{A}(x y)=0\right.$ for all $y \in A\}$.

PROOF. See R.S. Doran and V.A. Belfi [1, p. 325].

LEMMA 2. Let $A$ be an algebra with the identity. Let $x, y \in A$. If at least two points of the set $\{x, y, x y, y x\}$ belong to $G(A)$, then $\{x, y, x y, y x\} \subseteq G(A)$.

PROOF. See W. Rudin [2, p. 259].

LEMMA 3. Let $A$ be a Banach algebra. If $A$ has only the trivial idempotents, then $\sigma_{A}(x)$ is a connected set in $\mathbf{C}$ for each $x \in A$.

PROOF. See W. Rudin [2, p. 247].

LEMMA 4. Let $A$ be a unital Banach algebra. Then $\operatorname{Rad}(A)=G(A)^{c}$ if and only if for each $x \in \operatorname{Fr}(G(A)), \sigma_{A}(x)=\{0\}$.

PROOF. $(\Rightarrow)$ Suppose that $\operatorname{Rad}(A)=G(A)^{c}$. Let $x \in \operatorname{Fr}(G(A))$. Since $G(A)$ is open in $A, x \in G(A)^{c}$. Hence $x \in \operatorname{Rad}(A)$ and so $\rho_{A}(x)=0$ by Lemma 1. Therefore $\sigma_{A}(x)=\{0\}$ because $\sigma_{A}(x)$ is a nonempty set in $\mathbf{C}$.

$(\Rightarrow)$ Suppose that $\sigma_{A}=\{0\}$ for each $x \in \operatorname{Fr}(G(A))$. Let $x \in \operatorname{Rad}(A)$. Then $\rho_{A}(x)=0$ by Lemma 1. We claim that $x \in G(A)^{c}$. Assume that $x \in G(A)$. Then $x y=e=y x$ for some $y \in A$, 
and hence $1=\rho_{A}(e)=\rho_{A}(x y) \leq \rho_{A}(x) \cdot \rho_{A}(y)=0$, which is impossible. Therefore $\operatorname{Rad}(A) \subseteq G(A)^{c}$. It suffices to show that $G(A)^{c} \subseteq \operatorname{Rad}(A)$. Let $x_{0} \in G(A)^{c}$. Since $\sigma_{A}\left(x_{0}\right)$ is nonempty and compact, we can choose $\lambda \in \sigma_{A}\left(x_{0}\right)$ such that $\lambda_{0} \in \operatorname{Fr}\left(\sigma_{A}\left(x_{0}\right)\right)$. Then $x_{0}-\lambda_{0} e \in \operatorname{Fr}(G(A))$ by continuity. First we will show that $\sigma_{A}\left(x_{0}\right)=\{0\}$. Let $\lambda \in \sigma_{A}\left(x_{0}\right)$, then $x_{0}-\lambda e=\left(x_{0}-\lambda_{0} e\right)-\left(\lambda-\lambda_{0}\right)$. Hence $\lambda-\lambda_{0} \in \sigma_{A}\left(x_{0}-\lambda_{0} e\right)=\{0\}$. Hence $\lambda=\lambda_{0}$. Since $x_{0} \in G(A)^{c}, \lambda_{0}=0$. Hence $\sigma_{a}\left(x_{0}\right)=\{0\}$ for all $x_{0} \in G(A)$. Let $y \in A$. We claim that $x_{0} y \in G(A)^{c}$ or $y x_{0} \in G(A)^{c}$. Assume otherwise. Then $\left\{x_{0} y, y x_{0}\right\} \subseteq G(A),\left\{x_{0}, y, x_{0} y, y x_{0}\right\} \subseteq G(A)$ by Lemma 2, which is impossible, because $x_{0} \in G(A)^{c}$. Hence $x_{0} y \in G(A)^{c}$ or $y x_{0} \in G(A)^{c}$. By the above argument, $\rho_{A}\left(x_{0} y\right)=0$ or $\rho_{A}\left(y x_{0}\right)=0$. Since $\rho_{A}(u \cdot v)=\rho_{A}(v \cdot u)$ for all $u, v \in A, \rho_{A}\left(x_{0} y\right)=0=\rho_{A}\left(y x_{0}\right)$. Since $y$ is an arbitrary element in $A$, by Lemma 1, $x_{0} \in \operatorname{Rad}(A)$. Therefore $G(A)^{c} \subseteq \operatorname{Rad}(A)$. Hence $\operatorname{Rad}(A)=G(A)^{c}$.

PROPOSITION 5. Let $A$ be a unital semisimple Banach algebra. If $A$ has only the trivial idempotents and $\sigma_{A}(x)$ is countable for each $x \in \operatorname{Fr}(G(A)), A \cong \mathbb{C}$.

PROOF. Let $x \in \operatorname{Fr}(G(A))$, then $\sigma_{A}(x)$ is a connected subset of $\mathbb{C}$ by Lemma 3. Since $\sigma_{A}(x)$ is a connected, separable metric space, $\sigma_{A}(x)$ has exactly one point or an uncountable number of points of C. By our hypothesis, $\sigma_{A}(x)$ has exactly one point. Hence $\sigma_{A}(x)=\{0\}$, because $x \in G(A)^{c}$. Therefore we know that $\sigma_{A}(x)=\{0\}$ for all $x \in \operatorname{Fr}(G(A))$. It follows that $\operatorname{Rad}(A)=G(A)^{c}$ by Lemma 4. Since $A$ is an semisimple, $G(A)^{c}=\{0\}$. By the Gelfand-Mazur theorem, $A \cong \mathrm{C}$.

COROLLARY 6. If $A$ is a unital Banach algebra with $G(A)^{c}=\{0\}$, then $A \cong \mathrm{C}$.

PROOF. By Proposition 5, it suffices to show that $A$ is simisimple and that $A$ has only the trivial idempotents and that $\sigma_{A}(x)$ is countable for each $x \in \operatorname{Fr}(G(A))$. Since $\{0\} \subseteq \operatorname{Rad}(A) \subseteq G(A)^{c}=\{0\}, \operatorname{Rad}(A)=\{0\} . \quad$ Let $x$ be an idempotent in $A$. Then $x(x-e)=0$. Hence $x \in G(A)^{c}$ or $x-e \in G(A)^{c}$, hence $x=0$ or $x=e$. Let $x \in \operatorname{Fr}(G(A))$. Then $x \in G(A)^{c}$, hence $x=0$ and so $\sigma_{A}(x)=\{0\}$; therefore $\sigma_{A}(x)$ is countable for each $x \in \operatorname{Fr}(G(A))$.

COROLLARY 7. If $A$ is a unital semisimple Banach algebra such that for each $x \in \operatorname{Fr}(G(A))$, $\sigma_{A}(x)=\{0\}$, then $A \cong \mathrm{C}$.

PROOF. By Lemma 4 , it follows.

COROLLARY 8. If $A$ is a $C^{*}$-algebra such that for each $x \in \operatorname{Fr}(G(A)), \sigma_{A}(x)=\{0\}$, then $A \cong \mathrm{C}$.

PROOF. Since $C^{*}$-algebra is a simisimple Banach algebra, it follows from Corollary 7 .

REMARK 9. We give another proof of Corollary 8.

Let $x \in \operatorname{Fr}(G(A))$. Since $A$ is $C^{*}$-algebra, $\rho_{A}\left(x x^{*}\right)=\left\|x x^{*}\right\|=\|x\|^{2}$. Since $x \in G(A)^{c} ; x x^{*} \in G(A)$ or $x^{*} x \in G(A)^{c}$ by Lemma 2. Hence by the continuity of involution *, $x x^{*} \in \operatorname{Fr}(G(A))$ or $x^{*} x \in \operatorname{Fr}(G(A))$. By our hypothesis, $\rho_{A}\left(x x^{*}\right)=0=\rho_{A}\left(x^{*} x\right)$. Therefore, $\|x\|^{2}=0$, i.e., $x=0$. Since $\operatorname{Fr}(G(A))=\{0\}$, we can deduce that $G(A)^{c}=\{0\}$. By the GelfandMazur theorem, $A \cong \mathbb{C}$.

\section{REFERENCES}

1. DORAN, R.S. and BELFI, V.A., Characterizations of $C^{*}$ algebras: The Gelfand-Naimark Theorems, Marcel Dekker, Inc., New York, (1986).

2. RUDIN, W., Functional Analysis, McGraw-Hill, New York, (1973). 


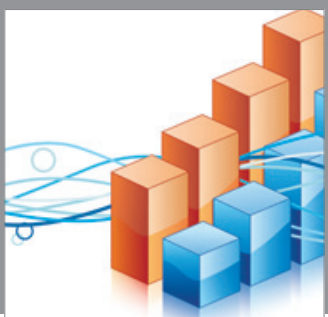

Advances in

Operations Research

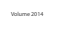

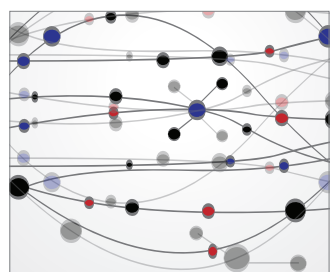

\section{The Scientific} World Journal
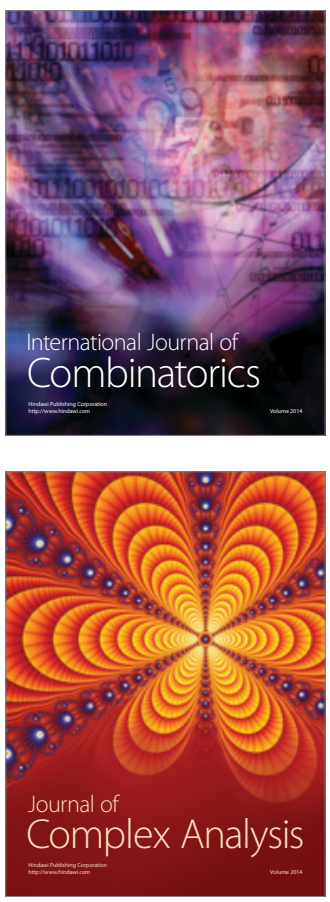

International Journal of

Mathematics and

Mathematical

Sciences
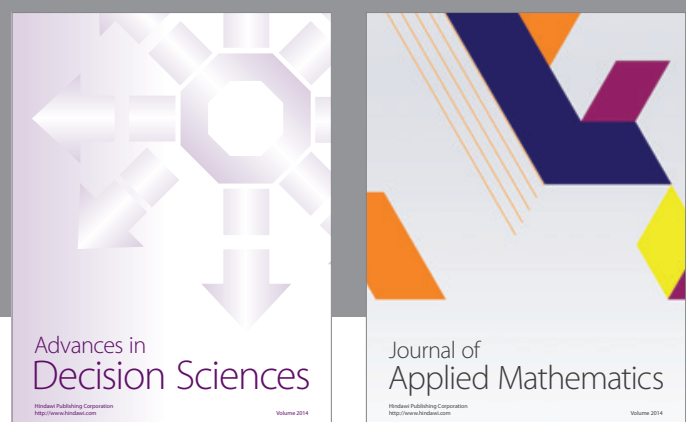

Journal of

Applied Mathematics
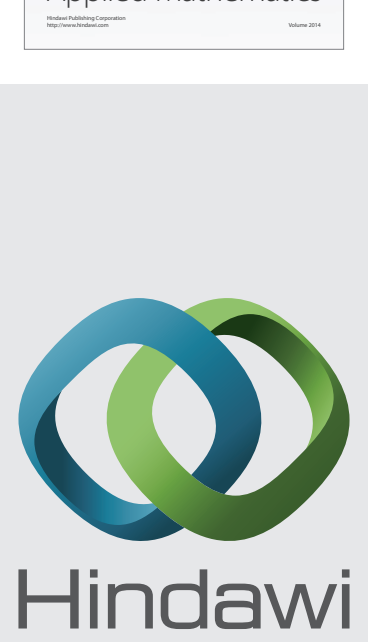

Submit your manuscripts at http://www.hindawi.com
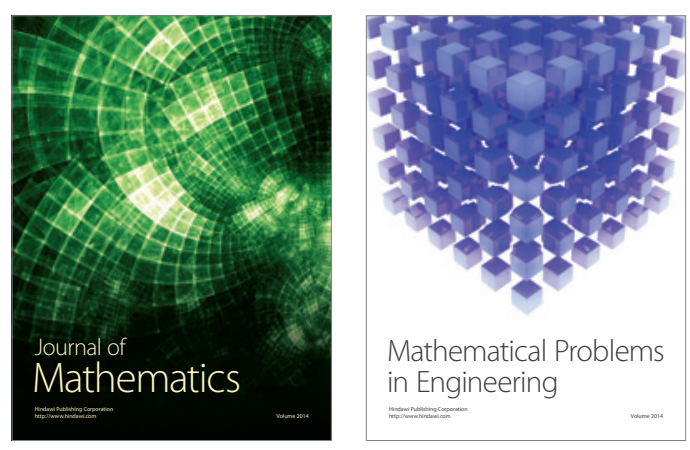

Mathematical Problems in Engineering
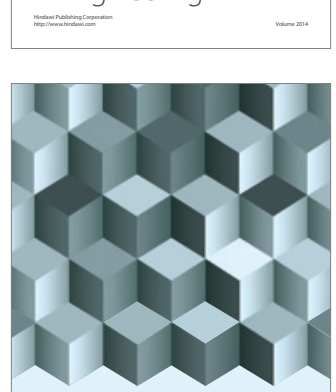

Journal of

Function Spaces
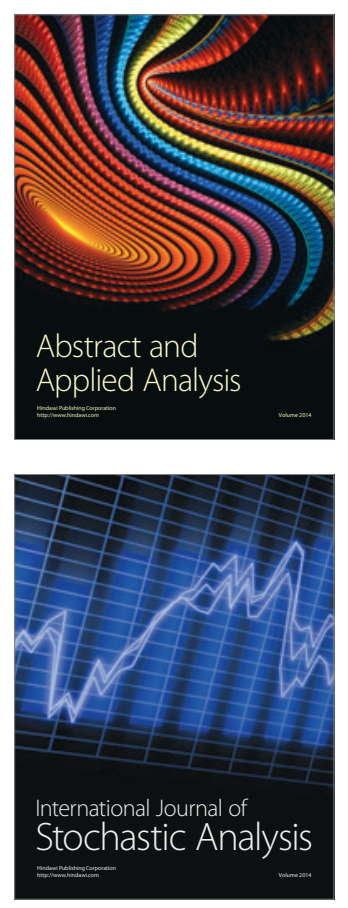

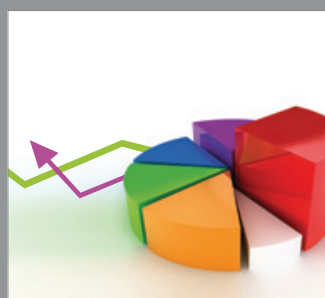

ournal of

Probability and Statistics

Promensencen
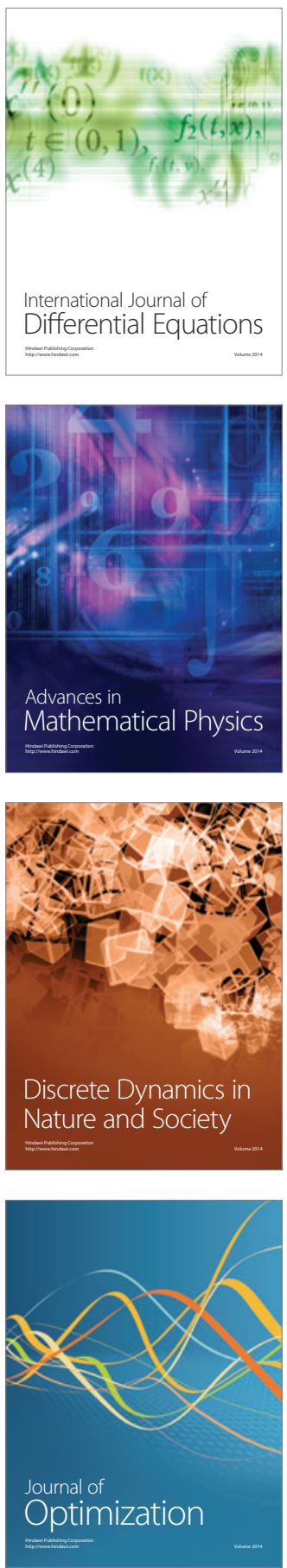